Contributions to Game Theory and Management, XIII, 427-440

\title{
Stochastic Data Transmission Model with Uncertainty on Dropper's Appearance *
}

\author{
Xue Juan ${ }^{1}$ and Elena M. Parilina ${ }^{2}$ \\ 1 Saint Petersburg State University, \\ 7/9 Universitetskaya nab., Saint Petersburg 199034, Russia \\ E-mail: juanxueqd@qq.com \\ 2 Saint Petersburg State University, \\ 7/9 Universitetskaya nab., Saint Petersburg 199034, Russia \\ E-mail: e.parilina@spbu.ru
}

WWW home page: http://www.apmath.spbu.ru/en/staff/parilina/index.html

\begin{abstract}
We consider a stochastic dynamic game with uncertainty about appearance of an dropper in infinite time horizon. For the lack of information about whether the dropper is present or not, two different states of nature can appear with some given probabilities at each stage. We study the non-cooperative behavior of players, and Nash equilibrium is considered as an equilibrium concept. We investigate how limited information about the dropper's appearance impacts the anti-jamming strategy.
\end{abstract}

Keywords: stochastic game, throughput, secrecy, Nash equilibrium

\section{Introduction}

In wireless communications, it is important to ensure the data security between a transmitter and a receiver when facing interference. In (Mukherjee et al., 2014), a comprehensive survey about principles of physical layer security in multiuser wireless networks is given. There is a variety of works which study jamming problems (e.g., see Altman et al., 2010; Altman et al., 2011; Vadlamania et al., 2016).

In jamming problems, a transmitter would like to transmit a signal with a good quality, while a jammer tries to degrade the signal quality at the intended receiver. For the conflicting interests of players, game theory has been widely employed in the literature to model different jamming problems when we consider malicious interference (see Charilas and Panagopoulos, 2010; Wu et al., 2012; Slimeni et al., 2016). In (Garnaev et al., 2012), a jamming game in a dynamic setting for a slotted ALOHA-like network is considered. In (Altman et al., 2011), the effect of partially available information and correlation among sub-carriers on the user behavior is investigated. Zero-sum games are considered in the case when the user does not know how jamming efforts are distributed among sub-carriers and the user does not know the fading channels' gains with certainty. In (Garnaev et al., 2017), the influence of uncertainty about whether or not a jamming attack is applied to an OFDM scenario on the resulting anti-jamming strategy has been investigated.

We consider a stochastic dynamic game with uncertainty about the dropper's appearance in infinite time horizon and study a simple communication network represented in Figure 1. In this communication network, a transmitter (Alice) wishes to communicate with the intended receiver (Bob) both secretly and with sufficient

\footnotetext{
* The work of the second author was supported by the Russian Science Foundation under grant no. 17-11-01079.
} 


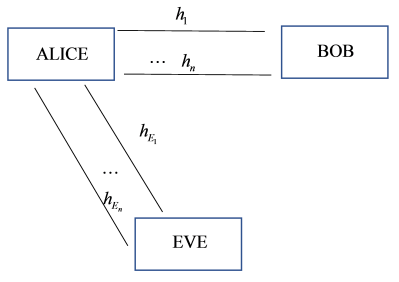

Fig. 1. Communication relationship between Alice, Bob and Eve

throughput. There is a jammer or a dropper (Eve) wants to eavesdrop on communication between Alice and Bob. The dropper Eve appears with uncertainty, and we take it into account by describing two different states of the game. In the state without dropper's appearance, the transmitter focuses solely on the reliability of communication. The utility is associated with communication reliability to be maintaining sufficient throughput which is reflected by Shannon capacity (Shannon, 1948). The state is called as a throughput state. In the state with dropper's appearance, the transmitter focuses on the secrecy of communication. We consider the secrecy capacity which is provided in (Csiszar and Korner, 1978) and also used in (Garnaev and Trappe, 2016). The state is called as a secrecy state.

A stochastic communication model is considered with infinite number of stages. At any stage, throughput and secrecy state can appear with a given probability. Payoff in a stochastic game is a random variable and the mathematical expectation of the player's payoff is assumed to be the utility of his payoff in stochastic game as in (Parilina and Tampieri, 2018). We study the non-cooperative behavior of the players, and calculate the Nash equilibria.

The paper is organized as follows. In the second section, we introduce the stochastic communication model and consider a problem setting with a unique player. In this case, we find an optimal strategy of the player and consider the factors which have influence on it. In the third section, we introduce a stochastic communication game with two players. We calculate the Nash equilibrium and consider the factors influencing the equilibrium strategies. Finally, we briefly conclude in the forth section.

\section{Stochastic Communication Model}

\subsection{Model Introduction}

We introduce the model of communication model given in Figure 1. Alice wishes to communicate with her colleague Bob but faces the uncertainty with the appearance of a eavesdropper (Eve), who wants to get information that Alice sends to Bob. To describe the uncertainty of Eve's appearance, we consider two states of Nature in the communication model-with and without Eve's showup.

In the communication network, the underlying wireless medium has been channelized into $n$ separate channels (e.g, different subcarriers in an OFDM system). Thus, Alice communicates to Bob across $n$ (sub)channels, and the response of channel $i$ is represented by its coefficient $h_{i}, i \in\{1, \ldots, n\}$. In the state with Eve's appearance, she can eavesdrop on Alice's communication across a broadcast medium (as in a wireless setting), and the channels from Alice to Eve are represented by co- 
efficients $h_{E_{i}}, i \in\{1, \ldots, n\}$. We focus on the complete channel state model, which means that Alice knows both the Alice-Bob and Alice-Eve channel coefficients.

We describe two possible states of Nature defining the set of strategies and the payoff(cost) function for the players participating in the game.

We describe the strategy for Alice as a power allocation vector $P=\left(P_{1}, \ldots, P_{n}\right)$, where $P_{i} \in R_{+}$is the power transmitted through channel $i$, i.e. it is the vector describing how to distribute her transmission power through different channels. Denote by $\bar{P} \in R_{+}$the total power to transmit by Alice, then the relation between the total power and the power in each channel is as follows $\sum_{i=1}^{n} P_{i}=\bar{P}, P_{i} \in$ $[0, \bar{P}], \forall i=1,2, \ldots, n$. The set of Alice's feasible strategies is denoted by $\Omega$, where $\Omega=\left\{\left(P_{1}, \cdots, P_{n}\right): P_{i} \in[0, \bar{P}], \sum_{i=1}^{n} P_{i}=\bar{P}\right\}$.

Alice faces different problems in two different states mentioned above.

State $\Gamma^{1}$. In this state without Eve's appearance there is one player. Alice can focus solely on the throughput associated with the transmitted signal to Bob, which can be considered as a utility for this communication between Alice and Bob. Thus, we call the state a throughput state, and denote it as $\Gamma^{1}$. The capacity or payoff to Alice is described by function

$$
v^{T}\left(P^{T}\right)=\sum_{i=1}^{n} \ln \left(1+\frac{h_{i} P_{i}^{T}}{\sigma^{2}}\right),
$$

where $h_{i}$ is fading gains of the main channels and $\sigma$ is background noise of the main channels, and $P_{i}^{T}$ is a power of transmitting by channel $i$ in $\Gamma^{1}$ satisfying $\sum_{i=1}^{n} P_{i}^{T}=\bar{P}, P_{i}^{T} \in[0, \bar{P}], \forall i=1,2, \ldots, n$, and vector $P^{T}=\left(P_{1}^{T}, \ldots, P_{n}^{T}\right)$ is a strategy of Alice in state $\Gamma^{1}$.

A task for Alice in $\Gamma^{1}$ is to maintain throughput as high as possible, i.e., to find a strategy maximizing her transmission payoff given by

$$
\hat{P}^{T}=\arg \max _{P^{T} \in \Omega} v^{T}\left(P^{T}\right) .
$$

One can notice that the solution of the maximization problem exists.

State $\Gamma^{2}$. In this state Alice's communication can be dropped by Eve, and Alice focuses on maintaining the secrecy of her communication. Thus, we shall call such a mode as operating in the secrecy state, and denote it as $\Gamma^{2}$. We use secrecy capacity to characterize the payoff of Alice in secrecy mode. In the case of multichannel transmission, such as in OFDM, the secrecy capacity can be described as

$$
v^{S}\left(P^{S}\right)=\sum_{i=1}^{n}\left[\ln \left(1+\frac{h_{i} P_{i}^{S}}{\sigma^{2}}\right)-\ln \left(1+\frac{h_{E i} P_{i}^{S}}{\sigma_{E}^{2}}\right)\right],
$$

where $h_{E_{i}}$ is fading gains of the eavesdropper's channels, $\sigma_{E}$ is background noise of the eavesdropper's channels, and $P_{i}^{S}$ is a power of transmitting by channel $i$ in $\Gamma^{2}$ satisfying $\sum_{i=1}^{n} P_{i}^{S}=\bar{P}, P_{i}^{S} \in[0, \bar{P}], \forall i=1,2, \ldots, n$, and vector $P^{S}=\left(P_{1}^{S}, \ldots, P_{n}^{S}\right)$ is a strategy of Alice in state $\Gamma^{2}$.

Without loss of generality, we assume that $\frac{h_{i}}{\sigma^{2}} \geq \frac{h_{E_{i}}}{\sigma_{E}}$ for any $i$. This relation implies that Alice does not use any channels that would not have supported any 
secrecy in Alice-Bob's communication. Just for simplification of notation, we introduce the following auxiliary notations:

$$
h_{i}:=\frac{h_{i}}{\sigma^{2}}, \quad h_{E_{i}}:=\frac{h_{E_{i}}}{\sigma_{E}^{2}} .
$$

Then we can rewrite the Alice's payoff in $\Gamma^{1}$ as

$$
v^{T}\left(P^{T}\right)=\sum_{i=1}^{n} \ln \left(1+h_{i} P_{i}^{T}\right),
$$

and her payoff in $\Gamma^{2}$ as

$$
\begin{aligned}
v^{S}\left(P^{S}\right) & =\sum_{i=1}^{n}\left[\ln \left(1+h_{i} P_{i}^{S}\right)-\ln \left(1+h_{E_{i}} P_{i}^{S}\right)\right] \\
& =\sum_{i=1}^{n} \ln \left(\frac{1+h_{i} P_{i}^{S}}{1+h_{E_{i}} P_{i}^{S}}\right) \\
& =\sum_{i=1}^{n} \ln \left(1+\frac{\left(h_{i}-h_{E_{i}}\right) P_{i}^{S}}{1+h_{E_{i}} P_{i}^{S}}\right),
\end{aligned}
$$

and from (5) we obtain that $v^{S}\left(P^{S}\right) \geq 0$ for any $i$, as $h_{i} \geq h_{E_{i}}$, and the term in square brackets under the sum is the saved capacity when Alice' communication is dropped by Eve.

Denote the strategy under which Alice maximizes her secrecy capacity in $\Gamma^{2}$ by

$$
\hat{P}^{S}=\arg \max _{P^{S} \in \Omega} v^{S}\left(P^{S}\right) .
$$

We use the superscript $S$ or $T$ just to distinguish the strategies between different states, we use superscript $T$ of $P^{T}$ to signal the strategy in throughput mode, and $S$ of $P^{S}$ to signal the strategy in secrecy mode.

Now we consider a stochastic communication model with infinite number of stages. In each stage, throughput and secrecy states may appear with given probabilities. We use matrix of transition probabilities to describe stochastic process of transitions between two states, which is given as follows:

$$
\Pi=\left(\begin{array}{ll}
p_{11} & 1-p_{11} \\
p_{21} & 1-p_{21}
\end{array}\right),
$$

where $p_{11} \in[0,1]$ is the probability to transmit from the throughput state to the throughput state, $1-p_{11}$ is the probability to transmit from the throughput state to the secrecy state, $p_{21} \in[0,1]$ is the probability to transmit from the secrecy state to the throughput state, $1-p_{21}$ is the probability to transmit from the secrecy state to the secrecy state.

We assume that players use stationary strategies, which means that a player chooses her strategy in each stage depending only on which state is realized at this stage. The problem of Alice in the stochastic communication game is to determine how to distribute her total transmission power through different channels in different states with uncertainty about transitions from state to state. Thus, the strategy in repeated communication model is characterized as $P=\left(P^{T}, P^{S}\right)$, where $P^{T}=$ $\left(P_{1}^{T}, P_{2}^{T}, \ldots, P_{n}^{T}\right)$ and $P^{S}=\left(P_{1}^{S}, P_{2}^{S}, \ldots, P_{n}^{S}\right)$. 
Definition 1. Stochastic communication problem $G$ is defined as follows:

$$
G=\left\langle\delta, \pi^{0}, \Pi, P, V\right\rangle,
$$

where $\delta$ is the discount factor, $\pi^{0}=\left(p_{0}, 1-p_{0}\right)$ is the the vector of the initial distribution on states $\Gamma^{1}$ and $\Gamma^{2}$, and $p_{0} \in[0,1]$ is the probability that state $\Gamma^{1}$ is realized at the first stage, $1-p_{0}$ is the probability that state $\Gamma^{2}$ is realized at the first stage, and $V=\left(v^{T}\left(P^{T}\right), v^{S}\left(P^{S}\right)\right)$ is a vector the Alice's payoffs in two states defined above.

The payoff to the player in problem $G$ is a random variable, and the mathematical expectation is used to represent the payoff, which can be written in the form (see Parilina, 2015; Parilina and Tampieri, 2018):

$$
E(P)=\pi^{0}(\mathbb{I}-\delta \Pi)^{-1} V .
$$

Now we illustrate the calculation process of the payoff when the game process starts from $\pi^{0}=(1,0)$. In this case, the payoff is expressed as follows:

$$
\begin{aligned}
E(P) & =E\left(P^{T}, P^{S}\right) \\
& =(1,0)\left(\begin{array}{cc}
1-\delta p_{11} & -\delta\left(1-p_{11}\right) \\
-\delta p_{21} & 1-\delta\left(1-p_{21}\right)
\end{array}\right)^{-1}\left(\begin{array}{c}
\sum_{i=1}^{n} \ln \left(1+h_{i} P_{i}^{T}\right) \\
\sum_{i=1}^{n}\left(\ln \left(1+h_{i} P_{i}^{S}\right)-\ln \left(1+h_{E i} P_{i}^{S}\right)\right)
\end{array}\right) \\
& =\frac{\left(1-\delta\left(1-p_{21}\right)\right) \sum_{i=1}^{n} \ln \left(1+h_{i} P_{i}^{T}\right)}{(1-\delta)\left(1-\delta\left(p_{11}-p_{21}\right)\right)} \\
& +\frac{\delta\left(1-p_{11}\right) \sum_{i=1}^{n}\left(\ln \left(1+h_{i} P_{i}^{S}\right)-\ln \left(1+h_{E_{i}} P_{i}^{S}\right)\right)}{(1-\delta)\left(1-\delta\left(p_{11}-p_{21}\right)\right)}
\end{aligned}
$$

Theorem 1. The optimal solution strategy $\left(P^{T}, P^{S}\right)$ in stochastic communication problem $G$ defined by (7) is the solution of the following system:

$$
\begin{aligned}
\frac{\partial L_{w}\left(P^{T}, P^{S}\right)}{\partial P_{i}^{T}} & =\frac{1-\delta\left(1-p_{21}\right)}{(1-\delta)\left(1-\delta\left(p_{11}-p_{21}\right)\right)} \frac{h_{i}}{1+h_{i} P_{i}^{T}}-w_{1}=0, i=1, \ldots, n \\
\frac{\partial L_{w}\left(P^{T}, P^{S}\right)}{\partial P_{i}^{S}} & =\frac{\delta\left(1-p_{11}\right)}{(1-\delta)\left(1-\delta\left(p_{11}-p_{21}\right)\right)}\left[\frac{h_{i}}{1+h_{i} P_{i}^{S}}-\frac{h_{E_{i}}}{1+h_{E_{i}} P_{i}^{S}}\right]-w_{2}=0 \\
i & =1, \ldots, n \\
\bar{P}-\sum_{i=1}^{n} P_{i}^{T} & =0 \\
\bar{P}-\sum_{i=1}^{n} P_{i}^{S} & =0
\end{aligned}
$$

Proof. The proof is given by the formulation of Karush-Kuhn-Tucker (KKT) problem. To find optimal strategy $P$ that maximizes the function $E(P)$ given above, we 
define the Lagrange function taking into account the power constraints:

$$
\begin{aligned}
L_{w}\left(P^{T}, P^{S}\right)= & \frac{\left(1-\delta\left(1-p_{21}\right)\right) \sum_{i=1}^{n} \ln \left(1+h_{i} P_{i}^{T}\right)}{(1-\delta)\left(1-\delta\left(p_{11}-p_{21}\right)\right)} \\
& +\frac{\delta\left(1-p_{11}\right) \sum_{i=1}^{n}\left(\ln \left(1+h_{i} P_{i}^{S}\right)-\ln \left(1+h_{E_{i}} P_{i}^{S}\right)\right)}{(1-\delta)\left(1-\delta\left(p_{11}-p_{21}\right)\right)} \\
& +w_{1}\left(\bar{P}-\sum_{i=1}^{n} P_{i}^{T}\right)+w_{2}\left(\bar{P}-\sum_{i=1}^{n} P_{i}^{S}\right) .
\end{aligned}
$$

The optimal strategy $P=\left(P^{T}, P^{S}\right)$ is found as a solution of the system (7)-(10) which are the conditions given by KKT method.

The system is nonlinear, and we provide a numerical solution. Suppose that Alice communicates to Bob across 2 (sub)channels, and we take the coefficient values of channels as $h_{1}=19, h_{2}=10, h_{E_{1}}=10, h_{E_{2}}=5$, the value of total power transmitted by Alice is $\bar{P}=100$, the transmission probability from state $\Gamma^{1}$ to $\Gamma^{1}$ is $p_{11}=0.3$, the transmission probability from $\Gamma^{2}$ to $\Gamma^{1}$ is $p_{21}=0.6$, the value of discount factor is $\delta=0.9$, take the vector of initial distribution as $\pi^{0}=$ $(1,0)$ to start from state $\Gamma^{1}$. By solving this system,we can get the unique optimal solution of the system $(7)-(10)$ which is $P=((50.0237,49.9763),(40.7829,59.2171))$. One can mention that according to the optimal Alice's strategy in state $\Gamma^{1}$ the transmission powers are almost equally distributed, but in a secrecy state the power of transmission via channel 2 is grater than via channel 1 .

\subsection{Sensitivity Analysis for Stochastic Communication Problem}

In this section, we illustrated how the Alice's optimal strategy in problem $G$ is influenced by some factors, including the total power transmitted by Alice and the characteristic of the communication network such as fading gains of the channels. By giving a network with three communication (sub)channels, we change one of the values of factors mentioned above to see how the strategies change when other parameters remain the same. For comfortable observation we use the number of percentage of total power in each channel as a dependent variable shown in the ordinate axis in any figure.

First, we explore the relationship of channel coefficient $h_{i}$ and other variables. From Figure 2, Figure 3 and Figure 4, we can see that both percentages of $P_{i}^{T}$ and $P_{i}^{S}$ increase with the increase of their channel coefficient $h_{i}$, and for any $P_{j}^{T}$ and $P_{j}^{S}$ with $j \neq i$, the percentages decrease when $h_{i}$ increases.

Then, we explore the relationship between $h_{E_{i}}$ and other variables. Taking into account that $h_{E_{i}}$ describes the channel coefficient with Alice and Eve, then the change of $h_{E_{i}}$ only influences the strategy in secrecy mode, that is the value $P_{i}^{S}$. From Figure 5, we can see that $P_{i}^{S}$ decreases with the increase of the eavesdropper's channel coefficient $h_{E_{i}}$, and for any $P_{j}^{S}$ with $j \neq i$ increases when $h_{E_{i}}$ increases.

Finally, we explore the relationship of total power $\bar{P}$ and other variables. From Figure 6 , we can see the tendency of increase for $P_{3}^{T}$ and decrease the others in throughput mode, and the change of power in the secrecy mode is not that obvious. From the coefficient $h=(20,22,10)$ and $h_{E}=(19,20,8)$, we can see that the value 
for the third channel is much smaller than the others. This may explain why Alice increases the power in this channel for throughput mode, just to get a balanced distribution among all channels.

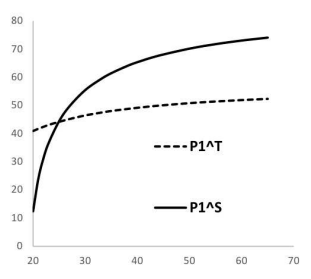

(a) Power changes in the first(b) Power changes in the secchannel.

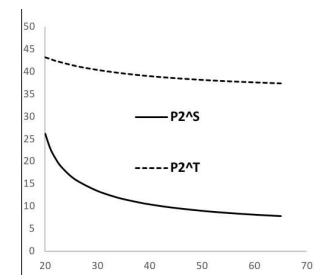
ond channel.

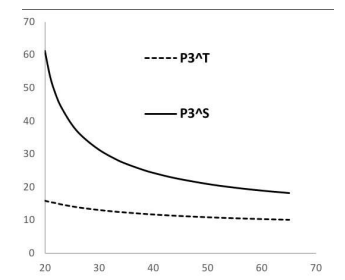

(c) Power changes in the third channel.

Fig. 2. The relationship between $h_{1}$ and power percentage in any channel.

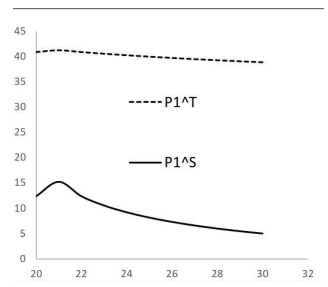

(a) Power changes in the first(b) Power changes in the secchannel.

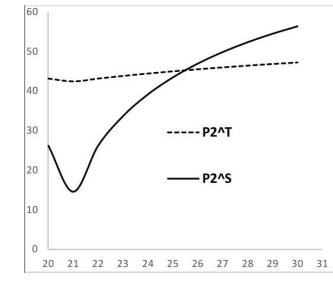

ond channel.

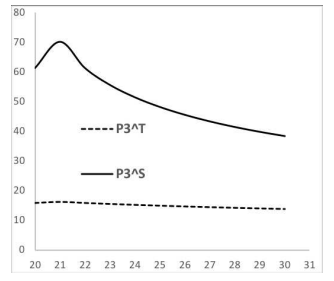

(c) Power changes in the third channel.

Fig. 3. The relationship between $h_{2}$ and power percentage in any channel.

\section{Two-player Stochastic game}

\subsection{Model}

Now suppose that the dropper Eve can participate in communication acting as a player in the game. We define stochastic game with two players with throughput and secrecy states that can appear with given probabilities in each stage.

In state $\Gamma^{1}$, Eve has no strategies, hence the Eve's payoff equals zero, i.e, $v_{E v e}^{T}(\cdot)=0$ for any Alice's choice. The strategy and payoff for Alice remains the same as in the last section. We use vector $P^{T}=\left(P_{1}^{T}, \cdots, P_{n}^{T}\right)$ to describe Alice's strategy as a power allocation vector, where $P_{i}^{T} \in R_{+}$is the power transmitted through channel $i$. All feasible strategies for Alice is denoted by set $\Omega$, $\Omega=\left\{\left(P_{1}^{T}, \cdots, P_{n}^{T}\right): P_{i}^{T} \in[0, \bar{P}], \sum_{i=1}^{n} P_{i}^{T}=\bar{P}\right\}$, where $\bar{P} \in R_{+}$is the total power to transmit by Alice.

The payoff to Alice in this state is described by function

$$
v_{\text {Alice }}^{T}\left(P^{T}\right)=\sum_{i=1}^{n} \ln \left(1+h_{i} P_{i}^{T}\right),
$$




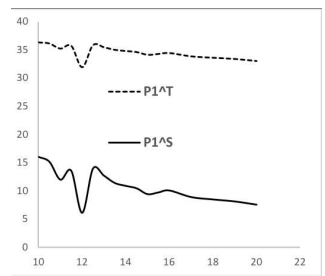

(a) Power changes in the first(b) Power changes in the secchannel.

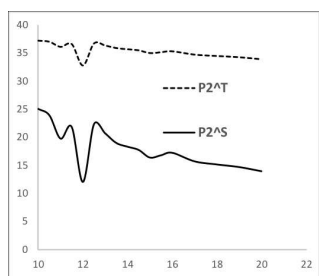
ond channel.

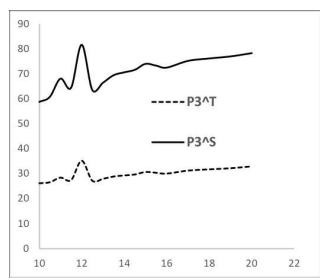

(c) Power changes in the third channel.

Fig. 4. The relationship between $h_{3}$ and power percentage in any channel.

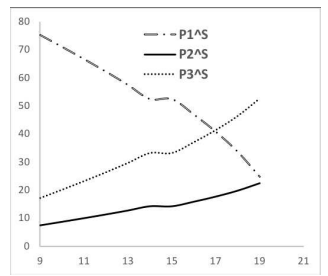

(a) Change of $P_{i}^{S}$ with $h_{E_{1}}$

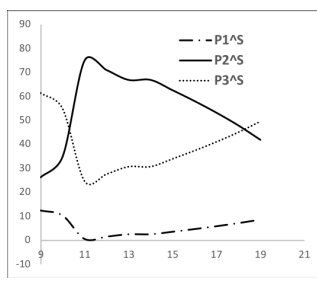

(b) Change of $P_{i}^{S}$ with $h_{E_{2}}$

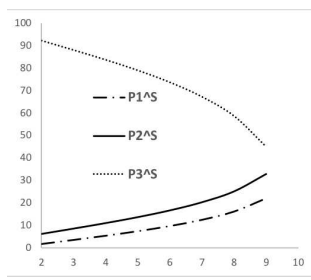

(c) Change of $P_{i}^{S}$ with $h_{E_{3}}$

Fig. 5. The relationship between $h_{E_{i}}$ and power percentage in the secrecy mode in any channel.

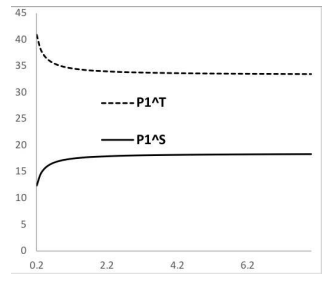

(a) Power changes in the first(b) Power changes in the secchannel.

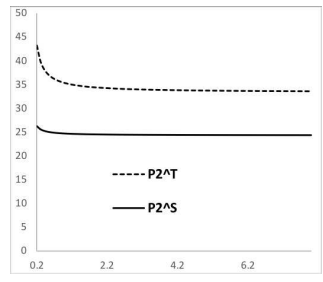
ond channel.

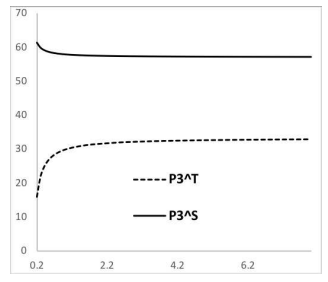

(c) Power changes in the third channel.

Fig. 6. The relationship between total power $\bar{P}$ and power percentage in any channel.

where $h_{i}$ is defined by $(3)$, strategy $P^{T}=\left(P_{1}^{T}, \cdots, P_{n}^{T}\right) \in \Omega$.

In state $\Gamma^{2}$, Eve has to decide how to distribute her total transmit power $\bar{Q}$, which is represented by vector $Q=\left(Q_{1}, \cdots, Q_{n}\right)$, where $Q_{i}$ is the power transmitted by Eve through channel $i$. Obviously, the relation between the total power and the power in each channel satisfies $\sum_{i=1}^{n} Q_{i}=\bar{Q}, Q_{i} \in[0, \bar{Q}]$ for any $i=1,2, \ldots, n$. Let $\Psi$ be the set of all feasible strategies of Eve, where $\Psi=\left\{\left(Q_{1}, \ldots, Q_{n}\right): Q_{i} \in\right.$ 
$\left.[0, \bar{Q}], \sum_{i=1}^{n} Q_{i}=\bar{Q}\right\}$. The payoff to Eve in this state is described by

$$
v_{\text {Eve }}^{S}\left(P^{S}, Q\right)=\sum_{i=1}^{n} Q_{i} \ln \left(1+h_{E i} P_{i}^{S}\right) .
$$

and the payoff to Alice equals

$$
v_{\text {Alice }}^{S}\left(P^{S}, Q\right)=\sum_{i=1}^{n}\left[\ln \left(1+h_{i} P_{i}^{S}\right)-\ln \left(1+h_{E_{i}} P_{i}^{S} Q_{i}\right)\right],
$$

where $P^{S}=\left(P_{1}^{S}, \ldots, P_{n}^{S}\right) \in \Omega$ is the Alice's strategy in state $\Gamma^{2}$ and $Q=$ $\left(Q_{1}, \ldots, Q_{n}\right) \in \Psi$ is Eve's strategy in this state.

A stochastic communication model is the game with infinite number of stages. In each stage, throughput or secrecy state may appear with some probability. The transition relationship between two states is defined by the matrix of transition probabilities given by (6).

Assuming that players use stationary strategies, the Alice's strategy in stochastic communication game is characterized by $P=\left(P^{T}, P^{S}\right)$, where $P^{T}=\left(P_{1}^{T}, \ldots, P_{n}^{T}\right)$ and $P^{S}=\left(P_{1}^{S}, \ldots, P_{n}^{S}\right)$. The Eve's strategy in the game is $Q=\left(Q_{1}, \ldots, Q_{n}\right)$.

Definition 2. Stochastic communication game $\bar{G}$ is defined by

$$
\bar{G}=\left\langle\delta, \pi^{0}, \Pi, P, Q, V_{\text {Alice }}, V_{\text {Eve }}\right\rangle,
$$

where $\delta \in(0,1)$ is the discount factor, $\pi^{0}=\left(p_{0}, 1-p_{0}\right)$ is the the vector of the initial distribution on states $\Gamma^{1}$ and $\Gamma^{2}$, and $p_{0} \in[0,1]$ is the probability that state $\Gamma^{1}$ is realized at the first stage, $1-p_{0}$ is the probability that state $\Gamma^{2}$ is realized at the first stage, $V_{\text {Alice }}=\left(v_{\text {Alice }}^{T}\left(P^{T}\right), v_{\text {Alice }}^{S}\left(P^{S}, Q\right)\right)$ is a vector composed by the Alice's payoff in both states, $V_{E v e}=\left(0, v_{E v e}^{S}\left(P^{S}, Q\right)\right)$ is a vector composed by the Eve's payoffs in both states.

The payoff to the player in game $\bar{G}$ is a random variable, and the mathematical expectation can be used to represent the payoff, which can be written in the following form (see Parilina and Tampieri, 2018):

$$
E_{\text {Alice }}(P, Q)=\pi^{0}(\mathbb{I}-\delta \Pi)^{-1} V_{\text {Alice }},
$$

and

$$
E_{E v e}(P, Q)=\pi^{0}(\mathbb{I}-\delta \Pi)^{-1} V_{E v e} .
$$

Definition 3. The Nash equilibrium in stochastic communication game $\bar{G}$ is the strategy profile $\left(\left(P^{T *}, P^{S *}\right), Q^{*}\right)$ such that

$$
\begin{aligned}
E_{\text {Alice }}\left(\left(P^{T *}, P^{S *}\right), Q^{*}\right) & \geq E_{\text {Alice }}\left(\left(P^{T}, P^{S}\right), Q^{*}\right) \text { for any } P^{T} \in \Omega, P^{S} \in \Omega, \\
E_{\text {Eve }}\left(\left(P^{T *}, P^{S *}\right), Q^{*}\right) & \geq E_{\text {Eve }}\left(\left(P^{T *}, P^{S *}\right), Q\right) \text { for any } Q \in \Psi .
\end{aligned}
$$

We describe the process of calculation of the Nash equilibrium in the game starting from state $\Gamma^{1}$, i.e., $\pi^{0}=(1,0)$. Making the similar calculations as in the last section, we find the Nash equilibrium strategy profile with players' payoff functions given by (13) and (14). The theoretical result giving the conditions for the Nash equilibrium in the following. 
Theorem 2. The Nash equilibrium $\left(\left(P^{T}, P^{S}\right), Q\right)$ in stochastic communication game $\bar{G}$ defined by (12) is given by the solution of the following system:

$$
\begin{aligned}
\frac{\partial L_{w}^{\text {Alice }}\left(P^{T}, P^{S}, Q\right)}{\partial P_{i}^{T}} & =\frac{1-\delta\left(1-p_{21}\right)}{(1-\delta)\left(1-\delta\left(p_{11}-p_{21}\right)\right)} \frac{h_{i}}{1+h_{i} P_{i}^{T}}-w_{1}=0, i=1, \ldots, n, \\
\frac{\partial L_{w}^{\text {Alice }}\left(P^{T}, P^{S}, Q\right)}{\partial P_{i}^{S}} & =\frac{\delta\left(1-p_{11}\right)}{(1-\delta)\left(1-\delta\left(p_{11}-p_{21}\right)\right)}\left(\frac{h_{i}}{1+h_{i} P_{i}^{S}}-\frac{h_{E_{i}} Q_{i}}{1+h_{E_{i}} P_{i}^{S} Q_{i}}\right) \\
& -w_{2}=0, i=1, \ldots, n, \\
\frac{\partial L_{w}^{E v e}\left(P^{S}, Q\right)}{\partial Q_{i}} & =\frac{\delta\left(1-p_{11}\right)}{(1-\delta)\left(1-\delta\left(p_{11}-p_{21}\right)\right)} \frac{h_{E_{i}} P_{i}^{S}}{1+h_{E_{i}} P_{i}^{S} Q_{i}}-w_{3}=0, \\
i & =1, \ldots, n, \\
\bar{P}-\sum_{i=1}^{n} P_{i}^{T} & =0, \\
\bar{P}-\sum_{i=1}^{n} P_{i}^{S} & =0 . \\
\bar{Q}-\sum_{i=1}^{n} Q_{i} & =0 .
\end{aligned}
$$

Proof. The proof is given by the formulation of Karush-Kuhn-Tucker (KKT) problem. To find the Nash equilibrium, i.e., strategy profile $\left(\left(P^{T}, P^{S}\right), Q\right)$, we define the Lagrange function taking into account the power constraints:

$$
\begin{aligned}
L_{w}^{\text {Alice }}\left(\left(P^{T}, P^{S}\right), Q\right)= & \frac{\left(1-\delta\left(1-p_{21}\right)\right) \sum_{i=1}^{n} \ln \left(1+h_{i} P_{i}^{T}\right)}{(1-\delta)\left(1-\delta\left(p_{11}-p_{21}\right)\right)} \\
& +\frac{\delta\left(1-p_{11}\right) \sum_{i=1}^{n}\left(\ln \left(1+h_{i} P_{i}^{S}\right)-\ln \left(1+h_{E_{i}} P_{i}^{S} Q_{i}\right)\right)}{(1-\delta)\left(1-\delta\left(p_{11}-p_{21}\right)\right)} \\
& +w_{1}\left(\bar{P}-\sum_{i=1}^{n} P_{i}^{T}\right)+w_{2}\left(\bar{P}-\sum_{i=1}^{n} P_{i}^{S}\right), \\
L_{w}^{\text {Eve }}\left(P^{S}, Q\right)= & \frac{\delta\left(1-p_{11}\right)}{(1-\delta)\left(1-\delta\left(p_{11}-p_{21}\right)\right)} \sum_{i=1}^{n} \ln \left(1+h_{E_{i}} P_{i}^{S} Q_{i}\right) \\
& +w_{3}\left(\bar{Q}-\sum_{i=1}^{n} Q_{i}\right) .
\end{aligned}
$$

The Nash equilibrium strategy profile $\left(\left(P^{T}, P^{S}\right), Q\right)$ is found as a solution of the system (15)-(20) which are the conditions given by KKT method.

The system is nonlinear, therefore, we provide the numerical simulation to demonstrate the solution of the system (15)-(20). Suppose that Alice communicates to Bob across 2 (sub)channels, and we take the coefficient values of channels as $h=(20,10)$, $h_{E}=(15,5)$, the value of total power transmitted by Alice is $\bar{P}=0.5$, the value 
of total power transmitted by Eve is $\bar{Q}=0.3$, the probability of transmission from state $\Gamma^{1}$ to $\Gamma^{1}$ is $p_{11}=0.3$, the probability of transmission from $\Gamma^{2}$ to $\Gamma^{1}$ is $p_{21}=0.6$, the value of discount factor is $\delta=0.9$, the vector of initial distribution over the set of states is $\pi^{0}=(1,0)$ (the game process is started from state $\left.\Gamma^{1}\right)$. By solving the system (15)-(20), we obtain the unique Nash equilibrium $P=((0.275,0.225),(0.172272,0.327728)), Q=(0.26164,0.0383604)$.

\subsection{Sensitivity Analysis for Stochastic Communication Game}

In this section, we explore the connection between different variables of the game described in the previous section. Given that the network with $n=2$, we illustrate the connection by examples represented in the figures.

First, we explore the relationship of the Alice's total power $\bar{P}$ and other variables. On Figure 7 one can see there is a significant change of Eve's power distribution, but only slight changes in Alice's strategy as a function of total power $\bar{P}$. More specifically, Eve decreases her power in the first channel and increases her power in the second one in the Nash equilibrium. The values of the parameters are $h_{1}=20$, $h_{2}=10, h_{E_{1}}=15, h_{E_{2}}=5, \bar{Q}=0.3$. The coefficient between Alice and Eve in the first channel is much larger than in the second one, this means that Eve decreases the power in the channel with larger coefficient. We remind that in all graphs, in the $y$-axis, the power in percentage is given (i.e., power in the channel divided by the total power of a player).

Then, we explore the relationship of total power $\bar{Q}$ and other parameters. As Eve chooses strategy only in the secrecy mode, then the strategy for Alice in the throughput mode remains the same. From Figure 8, we can see that $P_{1}^{S}$ increases and $Q_{1}$ decreases with the increase of the Eve's total power. The values of other parameters are $h_{1}=20, h_{2}=10, h_{E_{1}}=15, h_{E_{2}}=5, \bar{P}=1$. The difference between Alice and Eve in the first channel is much larger than the second one, this means that Alice increases the power in the channel with larger coefficient, but Eve decreases it.

Then, we examine the relationship between the channel coefficient $h_{i}$ and other variables. From Figures 9 and 10, we can see that both $P_{i}^{T}$ and $P_{i}^{S}$ increase with the increase of their channel coefficient $h_{i}$, and for any $P_{j}^{T}$ and $P_{j}^{S}$ with $j \neq i$ decrease when $h_{i}$ increases. Also, there is a increase of the Eve's power in the channel $i$ with the increase of coefficient $h_{i}$.

Now, we explore the relationship of variable $h_{E_{i}}$ and other variables. Coefficient $h_{E_{i}}$ only influences the strategy only in the secrecy mode. From Figures 11 and 12 , we can see that $P_{i}^{S}$ decreases with the increase of the eavesdropper's channel coefficient $h_{E_{i}}$, and with increase for Eve's power in this channel. 

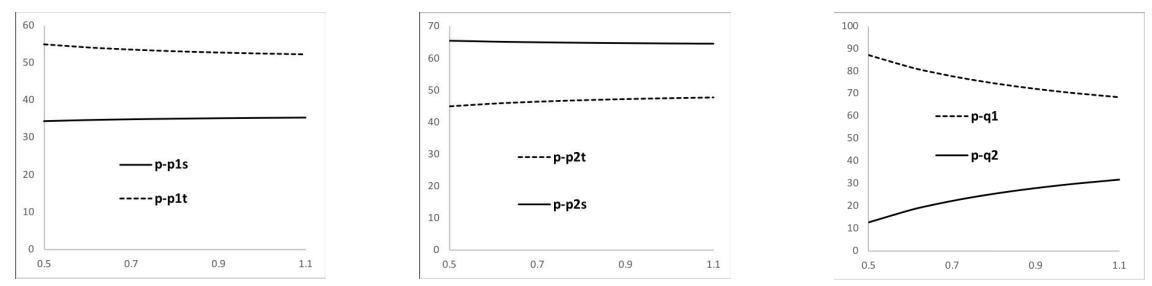

(a) Power changes in first(b) Power changes in second (c) Power changes in the channel for Alice. channel for Alice.

Eve's channel.

Fig. 7. The relation between $\bar{P}$ and the percentage of power distribution for both players.
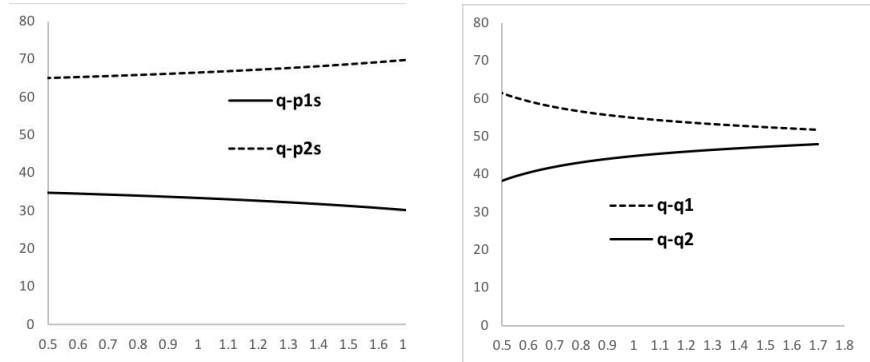

(a) Power changes for Alice's(b) Power changes in the Eve's channel in the secrecy state. channel.

Fig. 8. The relation between $\bar{Q}$ and the percentage of power distribution for both players.
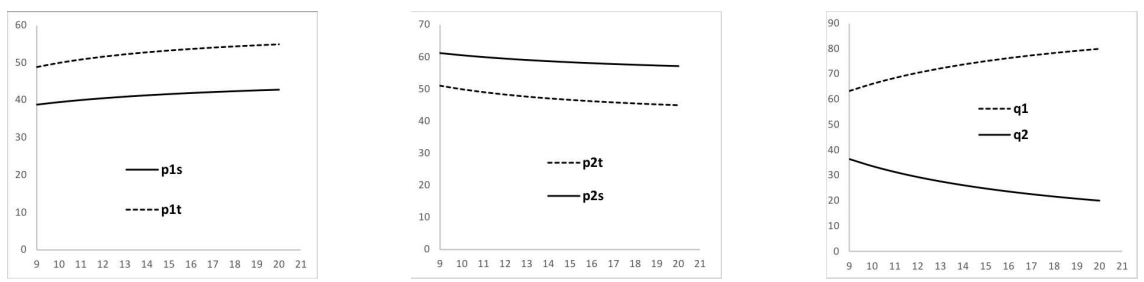

(a) Power changes in the Al-(b) Power changes in the Al- (c) Change of power in the ice's first channel. ice's second channel.

Eve's channel.

Fig. 9. The relationship between $h_{1}$ and the percentage of power distribution for both players. 

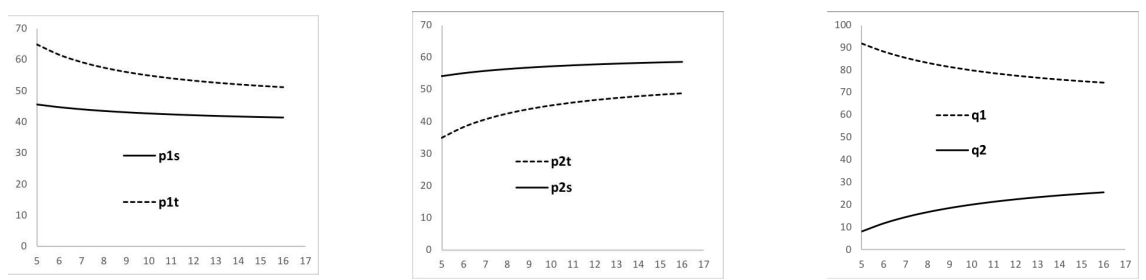

(a) Power changes in the Al-(b) Power changes in the Al- (c) Power changes in the ice's first channel. ice's second channel. Eve's channel.

Fig. 10. The relationship between $h_{2}$ and the percentage of power distribution for both players.
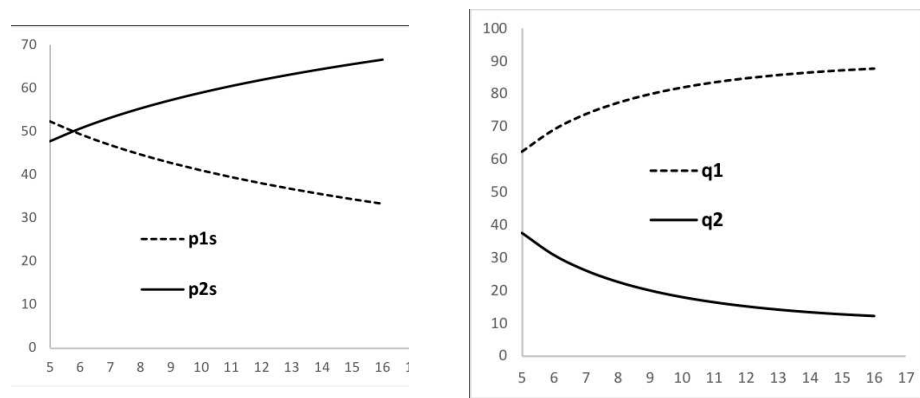

(a) Power changes for the Alice's channel in secrecy state.

(b) Power changes in Eve's channel.

Fig. 11. The relationship between $h_{E_{1}}$ and the percentage of power distribution for both players.
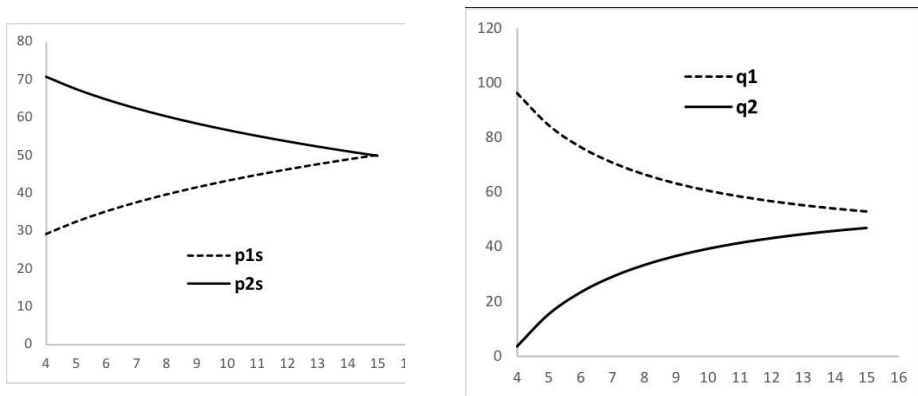

(a) Power changes for the Alice's channel in secrecy state.

(b) Power changes in the Eve's channel.

Fig. 12. The relationship between $h_{E_{2}}$ and the percentage of power distribution for both players. 


\section{Conclusion}

In this paper, the influence of uncertainty about dropper's appearance on the optimal strategy in stochastic communication problem and on the Nash equilibrium in stochastic communication game has been examined. Simulation results have been given to illustrate the relationship between some factors of the system and the optimal strategy and equilibrium strategies. In the future work, we can consider the influence of other features of the dropper like limited transmission power of the dropper on the optimal player's strategy.

\section{References}

Altman, E. Avrachenkov, K. and Garnaev, A. (2010). Fair resource allocation in wireless networks in the presence of a jammer. Performance Evaluation, 67(4), 338-349.

Altman, E. Avrachenkov, K. and Garnaev, A. (2011). Jamming in wireless networks under uncertainty. Mobile Networks and Applications, 16(2), 246-254.

Charilas, D. E. and Panagopoulos, A. D. (2010). A survey on game theory applications in wireless networks. Computer Networks, 54(18), 3421-3430.

Csiszar, I. and Korner, J. (1978). Broadcast channels with confidential messages. IEEE Transactions on Information Theory, 24(3), 339-348.

Garnaev, A., Hayel, Y., Altman, E. and Avrachenkov, K. (2012). Jamming game in a dynamic slotted ALOHA network. In: Game Theory for Networks (Jain, R. and Kannan, R. eds), Vol. 75, pp. 429-443. Springer.

Garnaev, A. and Trappe, W. (2016). Bargaining over the fair trade-off between secrecy and throughput in OFDM communications. IEEE Transactions on Information Forensics and Security, 12(1), 242-251.

Garnaev, A., Trappe, W. and Petropulu, A. (2017). Equilibrium strategies for an OFDM network that might be under a jamming attack. In: 2017 51st Annual Conference on Information Sciences and Systems (CISS), pp. 1-6. IEEE.

Mukherjee, A., Fakoorian, S. A. A. and Huang, J. (2014). Principles of physical layer security in multiuser wireless networks: A survey. IEEE Communications Surveys and Tutorials, 16(3), 1550-1573.

Parilina, E. M. (2015). Stable cooperation in stochastic games. Automation and remote control, 76(6), 1111-1122.

Parilina, E. M. and Tampieri, A. (2018). Stability and cooperative solution in stochastic games. Theory and Decision, 84(4), 601-625.

Shannon, C. E. (1948). A mathematical theory of communication. The Bell System Technical Journal, 27(3), 379-423.

Slimeni, F., Scheers, B. and Le Nir, V. (2016). Closed form expression of the saddle point in cognitive radio and jammer power allocation game. In: International Conference on Cognitive Radio Oriented Wireless Networks, pp. 29-40. Springer.

Vadlamania, S., Eksioglub, B., Medala, H. and Nandia, A. (2016). Jamming attacks on wireless networks: A taxonomic survey. International Journal of Production Economics, 172, 76-94.

Wu, Y., Wang, B., Liu, K. J.R. and Clancy, T.C. (2012). Anti-jamming games in multichannel cognitive radio networks. IEEE Journal on Selected Areas in Communications, $\mathbf{3 0}(\mathbf{1}), 4-15$. 